\title{
THE EARLY LESIONS IN VENO-OCCLUSIVE DISEASE OF THE LIVER
}

\author{
BY \\ G. A. STIRLING,* G. BRAS and A. E. URQUHART \\ Department of Pathology, University College of the West Indies, Jamaica
}

(RECEIVED FOR PUBLICATION APRIL 11, 1962)

A disease characterized by ascites and hepatomegaly was described in Jamaica by McFarlane and Branday in 1945, and later studied by Hill and his colleagues (Hill, Rhodes, Stafford and Aub, 1953), and by Bras, Jelliffe and Stuart (1954). Bras and his co-workers demonstrated occlusion of the small hepatic veins and accordingly named the condition 'Veno-occlusive disease of the liver' (V.O.D.). The exact nature of the occlusions has not been ascertained, although Bras and his colleagues likened some of them to an obliterating endophlebitis, and others to organized mural thrombi.

The death of three children early in the disease, one three days, another five days and a third 21 days after the onset of symptoms has prompted us to reinvestigate the pathogenesis of what are believed to be early lesions.

\section{Materials and Methods}

Formal-saline fixed liver tissue was available from all three cases. Paraffin sections were prepared in the usual way and stained with haematoxylin and eosin (H. and E.). Other staining methods used were Mallory's trichrome (M.T.), Mallory's phosphotungstic acid haematoxylin (M.P.A.H.), and Wilder's method for reticulin. One block from each case was sectioned serially, and initially every fifth section was stained. Other sections were stained when indicated.

Fresh material was available from the case dying at 21 days permitting the use of the immuno-histochemical techniques described by Coons, Creech, Jones and Berliner (1942), and Coons and Kaplan (1950). Blocks of liver tissue were frozen in a dry-ice alcohol mixture and stored at $-20^{\circ} \mathrm{C}$. Sections were cut in a cryostat at temperatures between $-20^{\circ}$ C. to $-10^{\circ} \mathrm{C}$. and mounted in the cold on glass slides. They were washed

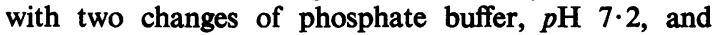
covered with rabbit anti-human fibrin serum conjugated with fluorescein isocyanate. After staining for 30 minutes they were washed repeatedly in the phosphate buffer and mounted in buffered glycerol $(p \mathrm{H} \mathrm{8.0)}$.

The anti-human fibrin serum was prepared by immunizing rabbits with human fibrin. The fibrin was

* Present address: King's College Hospital, London S.E.5. teased, cut into fine fragments, suspended in Freund's adjuvant and injected subcutaneously. Three doses of $1 \mathrm{~g}$. fibrin were given at intervals of 10 days, and the rabbits were bled three days after the last injection. The globulins were precipitated from the rabbit serum by saturation with anhydrous sodium-sulphate and the precipitate redissolved in distilled water and transferred to a dialysing bag and the sodium sulphate removed. The globulin was then coupled with fluorescein isocyanate and the labelled antiserum treated with powdered rat liver to reduce non-specific staining. The specificity of the antiserum was checked by demonstrating a single precipitation band in an Ouchterlony plate; by staining obvious fibrin deposits in placental tissue; and by treating duplicate sections with fluorescein-coupled antihuman fibrin serum absorbed with washed human fibrin. The sections were examined with the fluorescent microscope, photographed and then washed before restaining with H. and E. or M.T.

\section{Results}

The typical early lesion consisted of a network of delicate fibres partially occluding the centrilobular, sublobular and medium sized hepatic veins (Figs. 1, 2 and 3). These fibres appeared to arise from cells (Fig. 3), stained blue with M.T. and red to pink with M.P.A.H. and gave a negative immuno-histochemical reaction for fibrin (Fig. 4). Some, but not all, of the fibres were argyrophilic.

In one of the cases, studied only by ordinary staining techniques, clumps and strands of material staining as for fibrin were present in veins partially occluded by the obliterative lesion described above.

The walls of the affected veins were fragmented and thickened (Figs. 1, 2 and 3) and consisted not only of material staining as for collagen, but also aggregations of fibres staining as for fibrin, as well as material with a tinctorial reaction intermediate between fibrin and collagen. Wilder's method for reticulin revealed clumps and fibres of argyrophilic material. In the one case where fresh material was available the presence of fibrin was confirmed by the fluorescent antibody technique (Fig. 4).

There was marked congestion and laking of the 


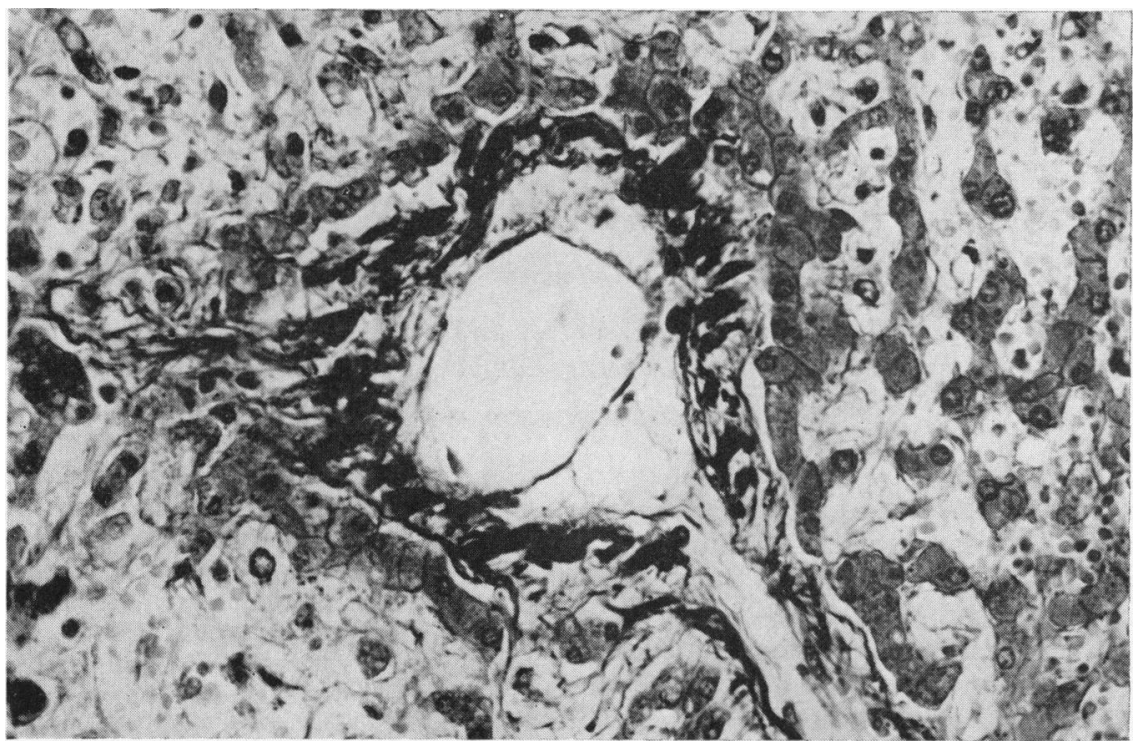

Fig. 1.-A sublobular vein partially occluded by a network of delicate fibres. Some of the dark staining material forming the wall of the vein is fibrin. (M.T. $\times 90$.)

blood in the centrilobular zones, and material staining as for fibrin surrounded the damaged veins and plugged some of the sinusoids. In the walls of larger veins dilated lymphatics were seen.

\section{Discussion}

There is no doubt that the fibrous occlusions in old cases of veno-occlusive disease developed from, and are essentially similar to, the delicate fibres seen in the early lesion and described here as the obliterative lesion. These fibres stained as for collagenous and reticular tissue and gave a negative reaction for fibrin with the fluorescent antibody technique. They were usually associated with cells but whether these

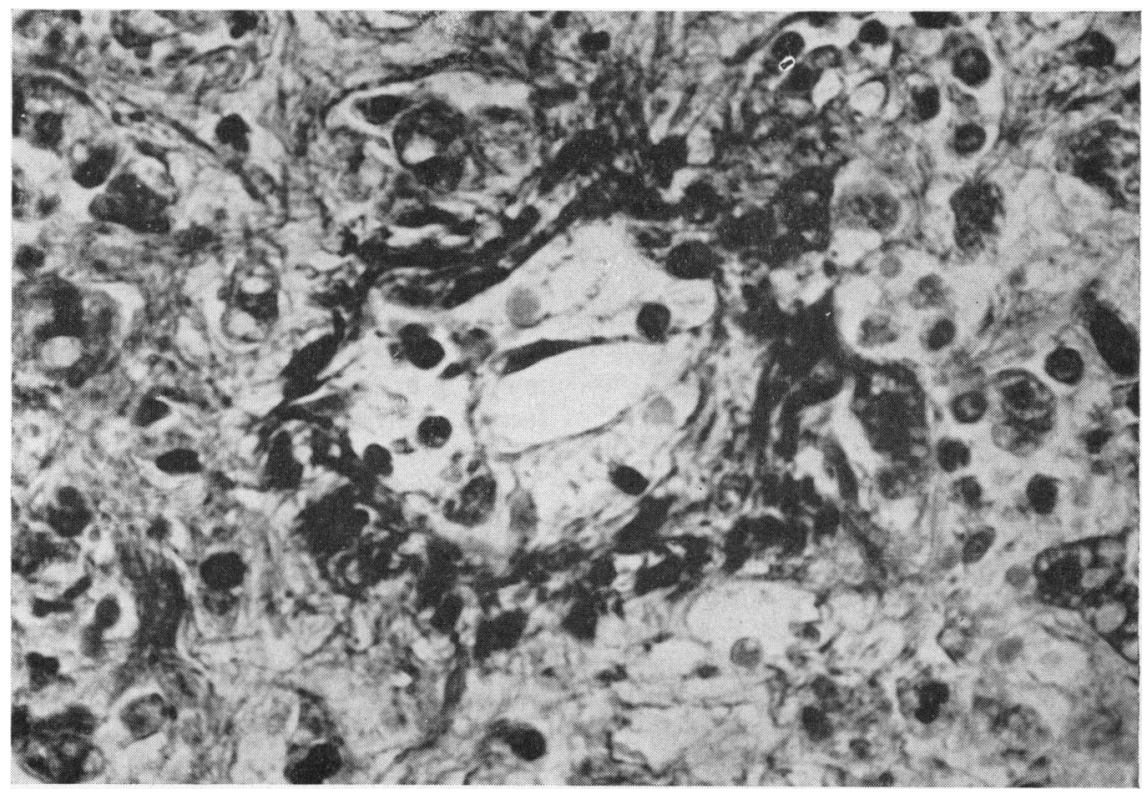

FIG. 2.-A centrilobular vein showing a similar lesion to that in Fig. 1. (M.T. $\times 187$.) 


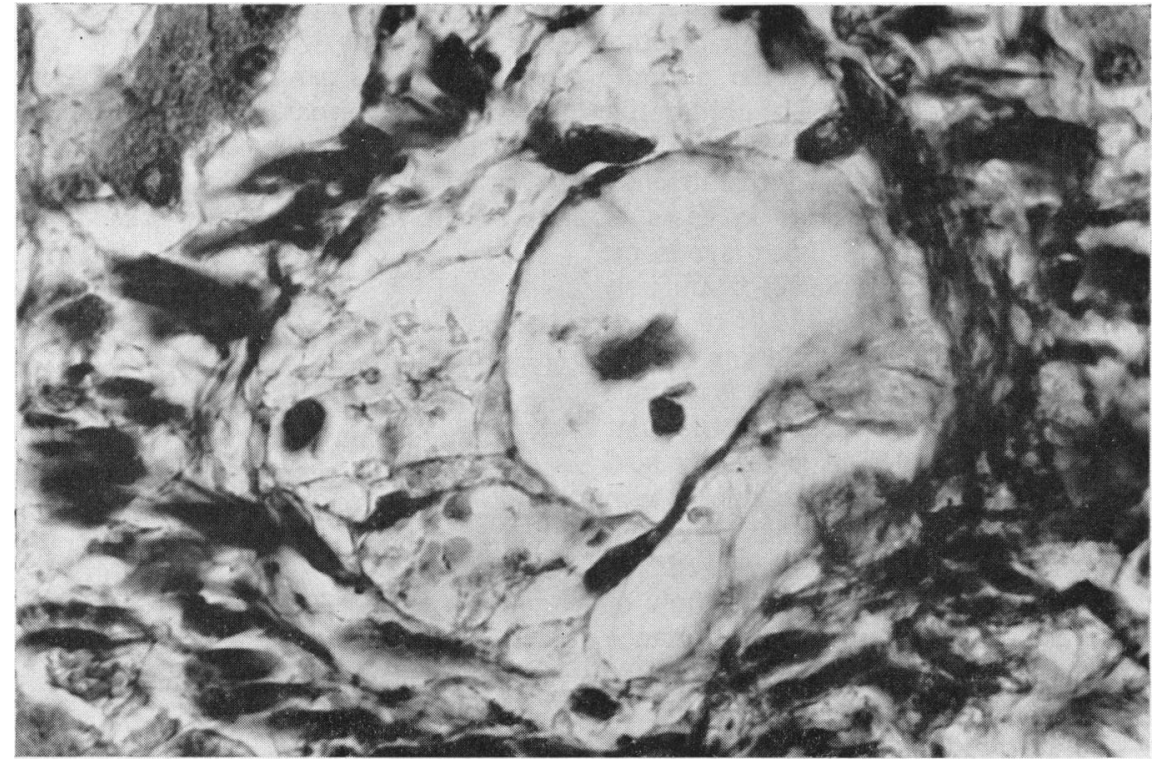

Fig. 3. - The lumen of a centrilobular vein partially occluded by a network of fibres apparently produced by cells. (M.T. $X 360$.)

were fibroblasts, endothelial cells or blood cells is not known. It is possible, of course, that the fibres represented aged fibrin which had lost its tinctorial and immunological identity, but we think this is unlikely since the cases died early in the course of the disease.

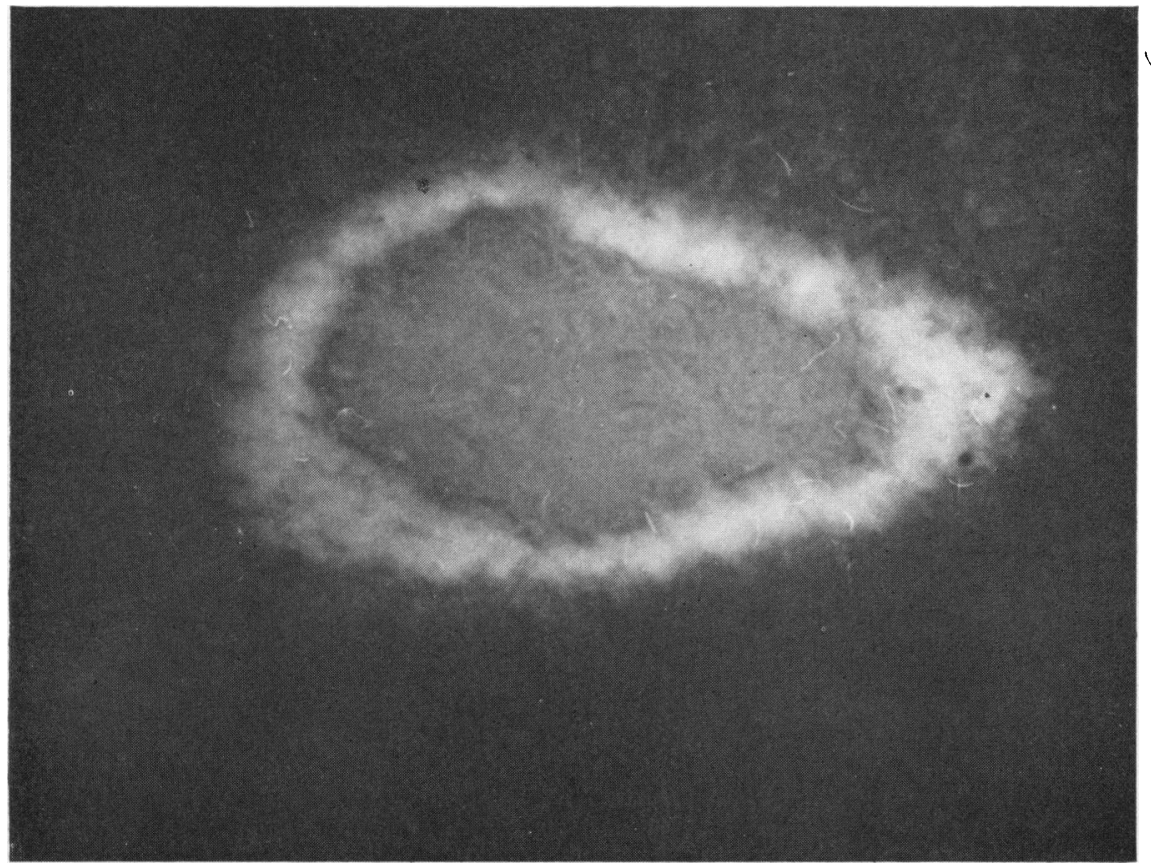

Fig. 4.-A centrilobular vein stained with fluorescence-labelled anti-fibrin serum shows specific fluorescence in the wall. The fibres in the lumen do not stain. (Ultraviolet light $\times 337$.) 
Thrombosis did not appear to be an important factor in veno-occlusive disease, although thrombi did form, as might be expected, in the partially occluded veins in one of our cases. The obliterative lesion in our material did not have the appearances or the staining reactions of recently formed thrombi so they differed from the occlusions in cases of Chiari's disease and the Budd Chiari syndrome which, according to Gibson (1960), 'manifestly developed from thrombi'.

A prominent feature in veno-occlusive disease and one described by Bras and Hill (1956) is massive centrilobular congestion with loss of centrilobular liver cells. The obliterative lesion is usually held responsible but the thickened, disorganized vein walls, the fibrin deposits surrounding the veins, and the collapse of the reticulin framework of the centrilobular zones, must also obstruct the flow of blood from the sinusoids. The relative importance of these lesions depends on the pathogenesis of veno-occlusive disease.

It is believed that veno-occlusive disease is caused by toxic substances present in bush teas used as herbal remedies. Injury of the hepatic veins by these substances may result in the obliterative lesion followed by massive centrilobular congestion and destruction of the centrilobular cells. An alternative pathogenesis is that the centrilobular cells are poisoned so that the blood 'lakes' in these zones, and fibrin is deposited in and about the walls of the hepatic veins. As a consequence of these changes the blood flow in the hepatic veins is reduced and the obliterative lesion develops in the manner described by Williams (1956) who showed that a reduction in blood flow could lead to obliterative endarteritis.
He also observed that in the early stages the obliterative endarteritis was reversible if blood flow was restored. This reversal of the obliterative process as well as the formation of new venous channels may help to explain the complete clinical recovery, reported by Stuart and Bras (1957), of some patients with acute veno-occlusive disease.

\section{Summary}

The occlusions in cases of veno-occlusive disease dying early in the disease were examined using conventional staining methods and the fluorescent antibody technique.

Thrombosis does not appear to be an important factor in veno-occlusive disease.

The pathogenesis of the obliterative lesion is discussed.

This work was supported by a grant from the Colonial Medical Research Grants Committee.

\section{REFERENCES}

Bras, G. and Hill, K. R. (1956). Veno-occlusive disease of the liver: essential pathology. Lancet, 2, 161 .

Jelliffe, D. B, and Stuart, K. L. (1954), Veno-occlusive disease of liver with nonportal type of cirrhosis, occurring in Jamaica. A.M.A. Arch. Path., 57, 285.

Coons, A. H., Creech, H. J., Jones, R. N. and Berliner, E. (1942). The demonstration of pneumococcal antigen in tissues by the use of fluorescent antibody. J. Immunol., 45, 159.

- and Kaplan, M. H. (1950). Localization of antigen in tissue cells. II. Improvements in a method for the detection of antigen by means of fluorescent antibody. J. exp. Med., 91, 1.

Gibson, J. B. (1960). Chiari's disease and the Budd-Chiari syndrome. J. Path. Bact., 79, 381.

Hill, K. R., Rhodes, K., Stafford, J. L. and Aub, R. (1953). Serous hepatosis: A pathogenesis of hepatic fibrosis in Jamaican children; preliminary report. Brit. med. J., 1, 117.

McFarlane, A. L. and Branday, W. J. (1945). Hepatic enlargement with ascites in children. ibid., $1,838$.

Stuart, K. L. and Bras, G. (1957). Veno-occlusive disease of the liver. Quart. J. Med., 26, 291.

Williams, G. (1956). Experimental studies in arterial ligation. J. Path. Bact., 72, 569. 